\title{
Virale Pneumonien mit ungewöhnlichen Folgen
}

Roberts C et al. Steroid-sensitive Post-Viral Inflammatory Pneumonitis (PVIP). Am J Respir Crit Care Med. 2010;182:1089-1090;

Jeon EJ et al. Acute eosinophilic pneumonia associated with 2009 influenza A (H1N1).

Thorax 2010;65:268-270

\section{Hintergrund}

Virale Pneumonien sind durch entzündliche Exsudate und zelluläre Infiltrationen gekennzeichnet. Diese Entzündungsreaktionen sind oftmals trotz einer antiviralen Therapie persistent.

Kürzlich wurde unabhängig voneinander über zwei Krankheitsbilder viraler Pneumonitiden berichtet, die gut auf Steroide ansprachen:

_ die steroidsensitive postvirale inflammatorische Pneumonitis (Fallbericht 1) und

_ die akute eosinophile Pneumonitis (Fallbericht 2).

\section{Fallbericht 1}

Catherine Roberts und Kollegen berichteten über drei Fälle einer Pneumonitis (zweimal durch H1N1-Influenzaviren, einmal durch Respiratory Syncytial Viren), die sich nach einer Hochdosis-Steroidtherapie (Methylprednisolon, $1 \mathrm{~g} / \mathrm{d}$, i.v.) rasch zurückbildeten und auf vorhergehende Behandlungen u.a. mit i.v.
Ribavirin nicht angesprochen hatten. Die Autoren bezeichneten diese mit schweren infiltrativen Veränderungen in der Computertomografie einhergehenden Erkrankungen als steroidsensitive postvirale inflammatorische Pneumonitis (Abb. 1).

Leider wurde keine bronch+oalveoläre Entzündungsdiagnostik oder eine andere weitergehende Abklärung durchgeführt.

\section{Fallbericht 2}

Sorgfältiger und unabhängig davon berichteten Eun Ju Jeon und Kollegen von einer akuten eosinophilen Pneumonie, assoziiert mit einer H1N1-Influenza-AInfektion. Die Viren wurden in der bronchoalveolären Lavage (BAL) nachgewiesen. Es bestand eine akute febrile eosinophile Pneumonie mit schwerer Hypoxämie, diffusen pulmonalen Infiltraten und einer massiven Erhöhung der BAL-Eosinophilen.

Differenzialdiagnosen, die als Ursache infrage kommen, wie eine Infektion mit oder eine hyperergische Reaktion auf Aspergillus, Coxsackie A2 oder Pseudomonas maltophilia, wurden ausgeschlossen, ebenso allergische Erkrankungen, Rauchen, Staub- oder Medikamentenexposition, Parasiten- und HIV-Infektionen.

Die ausgeprägte Eosinophilie in der BAL (Abb. 2) interpretierten die Autoren als akute eosinophile Pneumonie bei gleichzeitiger H1N1-Influenzainfektion.

\section{Kommentar}

Prof. Dr. med. Matthias Griese, München

Auch bei klinischen Pneumonien mit einem ungewöhnlich langem Verlauf ist ein Differenzialzellbild der BAL hilfreich und kann eine eosinophile Pneumonitis gut von der meist beobachteten neutrophilen Inflammationsreaktion abgrenzen. Obwohl eosinophile Infiltrate durch eine Untersuchung der Differenzialzytologie der BAL gut nicht invasiv untersucht werden können, erfolgt diese Untersuchung jedoch zu selten.

Virale Pneumonitiden gehen mit inflammatorischen Hypersensitivitätsreaktionen einher, die steroidsensitiv sein können. Eine probatorische, kurzzeitig hoch dosierte Steroidtherapie kann in Fällen, die mit den hier beschriebenen vergleichbar sind, hilfreich sein.

Weitere diagnostische und interventionelle Studien sind nötig, um eine klare allgemeine Vorgehensweise zu erarbeiten.

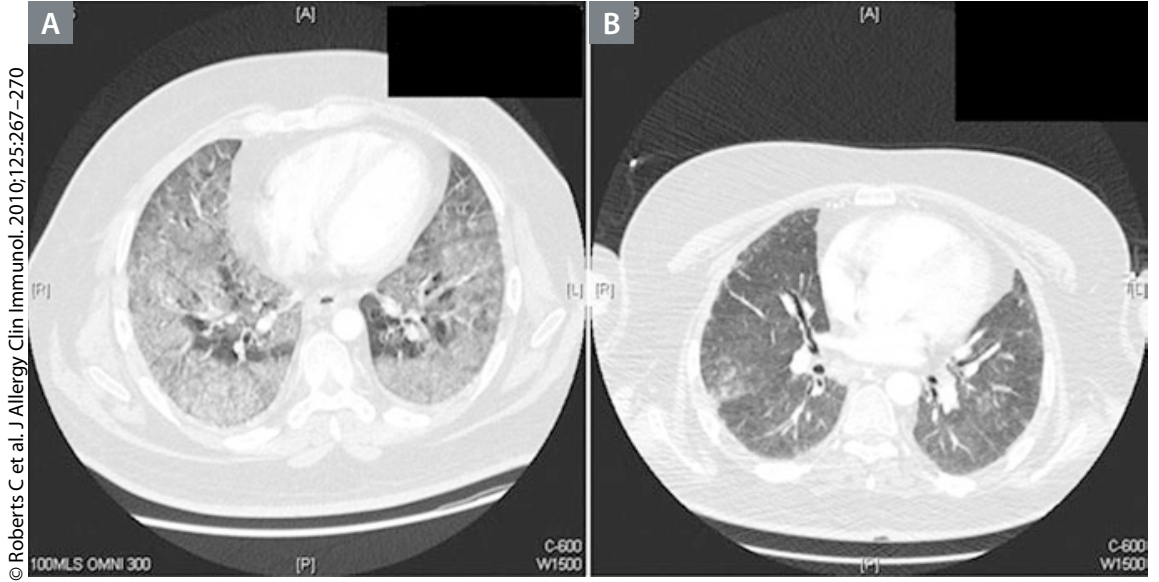

Abb. 1 Computertomografie einer Patientin mit H1N1-Pneumonitis bei der Aufnahme in die Klinik (A) und nach der Steroidtherapie (B).

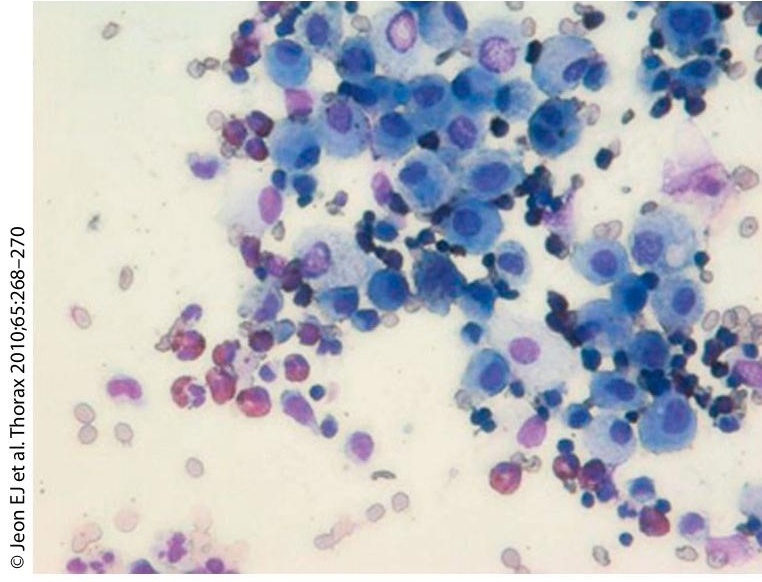

Abb. 2 Bronchoalveoläre Lavage: Eosinophilie nach H1N1-Influenza-A-Infektion. 